\title{
Wirelessly Accessible Sensor Populations (WASP) for Elderly Care Monitoring
}

\author{
Louis Atallah, Benny Lo, Guang-Zhong Yang \\ Department of Computing \\ Imperial College London, UK \\ \{latallah,benlo,gzy\}@doc.ic.ac.uk
}

\author{
Frank Siegemund \\ European Microsoft Innovation Center, EMIC \\ Aachen, Germany \\ franksie@microsoft.com
}

\begin{abstract}
This paper presents an application of a service-based architecture to pervasive monitoring of the elderly using ambient and wearable sensors. The design consideration of the model addresses heterogeneous computing and network resource utilization, allowing inter-operability and supporting dynamic environments to achieve system wide resource optimization. An application of this architecture is presented for assessing the activities of daily living, which is the basis for pervasive sensing for elderly care.
\end{abstract}

Keywords: Sensor networks; services; activity; elderly care

\section{INTRODUCTION}

Demographic changes associated with the aging population and the increasing numbers of elderly people living alone are leading to a significant change in the social and economic structure of our society. Advances in medicine and public health services have improved the overall longevity of all western nations, and it is expected that over the next 50 years, the proportion of people aged 60-plus around the world is expected to be doubled from the current $10 \%$ to $22 \%$ [1]. With the steady decline of the ratio of workers to retirees, a fundamental change in the way that elderly care is provided is inevitable. This change can be facilitated by the advances in the miniaturisation and cost reduction of sensor and embedded computing technologies, which would allow pervasive monitoring of the elderly to become a reality.

It is well known that even subtle changes in the behaviour of the elderly can give important signs of the onset and progression of certain diseases. Disturbed sleeping patterns could be caused, for example, by degenerative joint disease, gastro-oesophageal reflux along with congestive heart failure and chronic obstructive pulmonary disease. Changes in gait, on the other hand, can be associated with early signs of neurological abnormalities linked to several types of nonAlzheimer dementias. Subjects with neurologic gait abnormalities have a greater risk of developing dementia [2]. These examples highlight the importance of pervasive healthcare, i.e. continuous observation of behavioural changes in the elderly in order to detect deteriorations before a major event or exacerbation occurs.

In addition to behaviour monitoring, pervasive sensing could play an important role in chronic disease management and rehabilitation. Recent studies have looked into integrating miniature wearable sensors with orthoses, prostheses and

Permission to make digital or hard copies of all or part of this work for personal or classroom use is

granted without fee provided that copies are not made or distributed for profit or commercial advantage and that copies bear this notice and the full citation on the first page. To copy otherwise, to republish, to post on servers or to redistribute to lists, requires prior specific permission and/or a fee.

PERVASIVEHEALTH 2008, 30 Jan - 1 Feb. Tampere, Finland.

Copyright $\odot 2008$ ICST 978-963-9799-15-8

DOI 10.4108/ICST.PERVASIVEHEALTH2008.2777 mobility assistive devices [3,4]. Pervasive sensing is particularly appealing to these applications because it allows the implementation of closed-loop strategies based on increased complexity and flexibility of assistive and prosthetic devices.

Hitherto, several projects have investigated the use of pervasive sensors to provide a 'smart' environment for the observation of activities of daily living (ADL). Examples include Georgia Tech's ‘Aware Home' [5], Imperial College's UbiMon system [2] and SAPHE project [6], the Welfare Techno house in Japan [7] and MIT's PlaceLab [8]. However, the use of heterogeneous sensors, including both wearable and ambient sensors, in such large deployment projects poses a number of interesting challenges. These include dealing with energy constraints, memory and processing power restrictions, as well as privacy and security issues. In addition, embedding sensors in existing software environments is a major research issue on its right, involving sensor discovery, dealing with different communication protocols and maintenance.

The WASP (Wirelessly Accessible Sensor Populations) project aims to address these issues in order to provide a complete pervasive sensing system for practical applications. In particular, elderly care is one of the target applications of the WASP project. It aims to provide a pervasive framework by integrating ambient and wearable sensors for continuous monitoring of the elderly, facilitating risk analysis, and providing long term trend observation [9]. This article will highlight the WASP system architecture for elderly care and present a service model for implementing such a scenario.

\section{The ElDERly CARE General ScENARIO}

\section{A. System Architecture}

The general system architecture for the elderly care scenario is presented in Fig. 1. It outlines a simple, yet highly structured architecture to encapsulate different key aspects of the scenario. The proposed architecture is composed of:

The Body Sensor Network (BSN): A network of wireless sensors worn by (or implanted in) a person, which are designed to collect biomedical, physiological and activity data [10].

The Ambient Sensor Network (ASN): A network of wireless ambient sensors installed in or around the house, appliances and furniture, which are designed to collect different types of 
data, providing information on the patient's environment and activities.

The Personal Mobile Hub (PMH): This could be a mobile phone or a PDA (Personal Digital Assistant) and acts as the HUB of a Body Sensor Network. Apart from gathering the sensor data, it can also serve as an alarm trigger with a emergency press button. In addition, it can be used as a data logger when the user is out of the range of the ASN.

The Remote Data Collector (RDC): A server with a secured database which collects the events and data from the patients and homes via the WSN Hub through the internet, and also acts as a proxy for hospitals.

The Wireless Sensor Hub (WSN Hub): This is an abstract gateway that can collect data from the PMH or from the sensors directly and forward it to the RDC.

The Assistance Providers: An abstract term which is used to represent a generic care giver, such as a General Practitioner (GP), a nurse, or a person at the Remote Assistance Centre (RAC) that can provide assistance to the elderly.

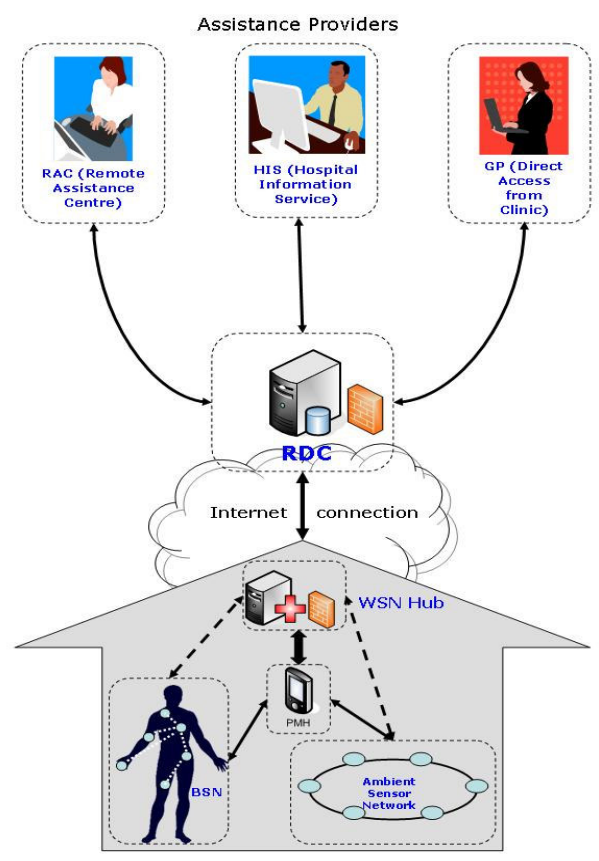

Figure 1. General system architecture illustrating the data flow for a typical WASP elderly care scenario.

\section{B. An Overview of Elderly Care Scenarios and Use Cases}

In order to identify key exemplar scenarios as part of the WASP project, more than 90 General Practitioners were contacted and interviews were conducted with patients, caretakers and stake-holders [9]. Both patient needs and system availability were taken into consideration and the analysis has led to the selection of 4 main representative elderly care scenarios. The following is a brief summary of these scenarios with selected use cases.

ADL monitoring using wearable and ambient sensors: ADL indices, such as activity levels, mobility, communication, eating and sleeping habits can be used by health professionals to assess the well-being of the elderly. Use cases for this scenario include the detection of hypothermia risks and sleep monitoring.

Social interaction monitoring using wearable and ambient sensors: A lack of social interaction can be used as a precursor to detect depression. Deviations from normal patterns can also provide tell-tale signs of the general mental health of the elderly. Use cases include the monitoring of activities and behaviours of a number of people in one location, such as an elderly care home.

Monitoring chronic diseases using wearable and ambient sensors: Effective chronic disease management is the basis for improving the quality of life of individuals with chronic conditions, as care programmes can be adjusted to the individual's needs, and early signs of deterioration could be detected. Use cases include:

- Report generation for chronic disease monitoring (comparing values across time and highlighting any major changes).

- Calculation of normal (or average) parameters of the elderly, such as the normal activity level, or oxygen saturation level.

- User activated alarms in case the patient feels that his/her general well-being is deteriorating.

- Automatic detection of an anomaly in the collected data and the triggering of an alarm.

Interoperability with implantable devices for long-term monitoring: The aim of this scenario is to provide a future outlook where the system presented can also be used for new generations of implantable devices which will have the functions, such as human power scavenging, self-configuring, self-managing and self-healing.

\section{A SERVICE MODEL FOR THE ELDERLY CARE SCENARIO}

\section{A. Why Services?}

The scenario explained above is an example of a heterogeneous sensor environment involving multiple sensors, gateways, mobile devices (the PMH) and a backend infrastructure. Such scenarios require the interoperation of a range of different computing devices. In addition, severe resource constraints of sensor nodes, as well as privacy and security concerns require an architecture that is self-optimised during execution by incorporating specific constraints and policies. A serviceoriented model provides a mechanism to access capabilities using a prescribed interface where constraints and policies are encapsulated in the service description. Therefore, a service model of a heterogeneous sensor environment should have the following four properties:

- Interfaces: comprise the functional attributes of a service and describe how to access the capabilities of a certain service. A mobile communication hub can retrieve interface descriptions about services provided 
by ambient sensors which would enable it to make use of these services in an ad-hoc fashion.

- Service Description: describes both functional aspects of a service as well as the extra-functional aspects. The latter includes, for example, information on the energy required to implement a service and the level of access allowed (privacy). These can be used for optimizing the performance of a sensor network.

- Interoperability: several industrial standards exist for service oriented architectures (especially for web services [11]), and adopting such standards will enable sensor nodes to interact with other computing devices.

- Constraints and Policies: these are regarded as part of the service description, and can specify energy, security and privacy issues. Information about constraints and policies can be used in a service composition process to find appropriate services and to apply optimization strategies.

In designing the service model for the WASP project, more than 10 service description standards have been evaluated and compared. The criteria for comparison include: storage models, the size of the description (and memory constraints), processing overhead, ability to interact with XML-based service descriptions, reliability, interoperability, interfacing and the ease of expressing constraints and policies, as well as other extra-functional aspects. Several service frameworks for sensors, traditional computer networks, and specific application areas were studied in depth [12]. A service model that covers all of these three areas is still largely missing although there are service frameworks that present efficient solutions for each of them. For example, the EPC standards like EPCIS and EPC [13] Application Level Events mainly focus on passive or semi-passive tagging technologies. Thus, energy consumption is not one of the main issues because reader devices (RFID readers, for example) are typically mains powered. IEEE 1451 [14] covers some of the problems in sensor networks, but it is relatively low level as it does not really consider heterogeneous environments, in which sensors must interact with other sensor nodes, mobile devices, or a backend infrastructure. Technologies like the Devices Profile for Web Services (DPWS), on the other hand, typically require relatively powerful sensor nodes. They also assume that a sensor would have a TCP/IP stack and an XML/SOAP parser which is often not available on the sensor nodes considered in this work. Finally, although SensorML and DPWS [12] allow the assignment of extra-functional aspects to sensor services, it has not been shown, so far, how this additional information can be used in a dynamic service composition process or during the static deployment of applications on sensor nodes.

In summary, although service-oriented architectures present a suitable vehicle for dealing with integration problems in heterogeneous sensor environments, existing approaches mainly focus either on low-level sensor nodes, passive tagging technologies or backend systems. The challenge of this work will be to integrate sensor nodes into existing service-oriented architectures (such as Web Services) considering the unique demands and resource constraints of wearable and ambient sensors.

\section{B. WASP Service Model}

Given the requirements of the scenarios, as well as previous work on service oriented models, a WASP service model was developed aiming for heterogeneous resources utilisation and optimisation whilst providing interoperability with backend systems and supporting dynamic interactions. Although the WASP model is based on concepts from other standards, such as expressing characteristics and constraints in metadata, as done in SensorML [12], several extensions are proposed where necessary to address specific elderly care requirements and provide optimisation options. The WASP service model (Fig. 2) is composed of the following:

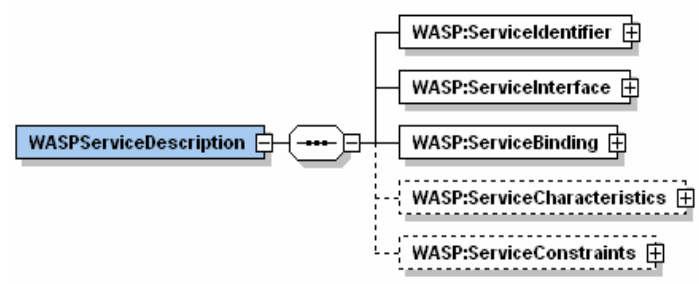

Figure 2. WASP service description model.

- Service Identifier: the service identifier contains information necessary to locate and address a specific service as well as the information required to retrieve further information about a service. It is assumed that a service is hosted on a particular device with a corresponding device address. The service identifier also contains description addresses that can be used to retrieve information about the service from the backend infrastructure Thus, only the service identifier must be stored on the sensor node itself, and the rest of the service description can be retrieved from a server in the backend.

- Service Interface: the service interface contains the functional aspects of a service, i.e., it specifies the syntax and order of messages in a service, and the involved data types.

- Service Binding: the service binding - or service grounding - specifies how the messages of a service are mapped to a concrete communication protocol. Service binding helps to deal with heterogeneity. An example is switching between web-services when communicating with a backend infrastructure and lightweight binding for resource-constrained sensor nodes.

- Service Characteristics: service characteristics define the core properties of a service and which facilitates resource optimisations. Example service characteristics that are used in the WASP description include: energy consumption, timing information, memory usage, processing overhead, reliability and security (shown in Fig. 3).

- Service Constraints: service constraints restrict the service composition process in that they specify under what conditions a service can be used. For example, a healthcare service that sends a patient's heart rate data 
over a network can specify that the data sent must be encrypted using a predefined algorithm.

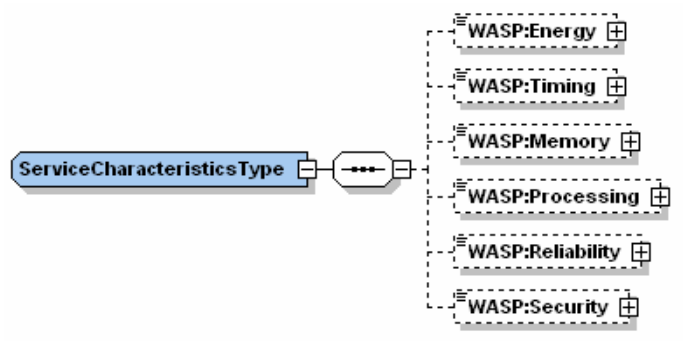

Figure 3. Service characteristic type for the WASP model.

\section{The Use of Services for the Elderly Care Scenario}

To describe the use of services in the elderly care scenario, the services were divided into two key dimensions: architectural (node, networking and background services) and functional (gateway, security, programming model and maintenance services). A list of services that can be easily expressed with the model mentioned above was considered for each dimension. For the architectural division, these services are:

- Node Services: examples of node services include returning node configurations, setting node parameters, varying transmission rates, running data compression on sensor data, and applying filters on the data.

- Networking Services: examples include service discovery, routing, topology and neighbour discovery, sending/receiving data from the BSN to the nearest gateway, and offloading data from the WSN hub to the RDC.

- Background Services: these can be divided into two groups. The first group provides a standardised interface for other business/enterprise applications to get access to sensor data, such as defining events of interest and data mapping using healthcare data standards (HL7). The second group helps in dealing with resource restrictions and balancing some of the sensors' workload to the backend infrastructure. Such services include history services allowing sensors to offload captured data to a backend server and the fusion of sensor data.

For the functional division, service categories are:

- Gateway services: gateway services mediate between the sensor nodes and the background infrastructure. Gateways typically have more resources than sensor nodes, and they can be either stationary or mobile. The $\mathrm{PMH}$ and the WSN hub are examples of gateways in Fig.1. Services include buffering data on the gateways for later transmission, pre-processing data, coordinating sensor nodes and mapping between different service models (on the node and the backend infrastructure).

- Security services: security services support secured and trusted sensor data processing from nodes to business programming services including gateway and network services. The major identified security requirements are availability, confidentiality, integrity and trustworthiness of data processing.

- Programming model services: these describe the services needed to support an event-based programming model and many of them can be considered as network or node services. Examples include scheduling event subscriptions, content-based querying and addressing, creating cluster topologies and priority-based communication.

- Maintenance services: the purpose of maintenance services is to monitor the operation of sensors and to alter the behaviour of embedded sensor devices, especially after deployment. Typically, maintenance services are only accessible to authorised persons and thus pose special security constraints. Examples of maintenance services include retrieving the current status of nodes (regarding energy, memory and processing), adding new components and reprogramming a sensor node.

The above division of services presents a methodical way of service description for a complex scenario which would help tackle some of the challenges in implementation which include: heterogeneity, interoperability, optimisation and the support of dynamic and static environments.

Coping with heterogeneous resources is tackled by using different bindings; a lightweight binding for sensor nodes and a web-services binding for the back-end systems. The expression of service characteristics and constraints in metadata is a way of allowing other parties to access this metadata and apply optimisations. Interoperability with backend systems is achieved by mapping the WASP service description to Web service standards. To deal with dynamic environments, WASP service descriptions contain a description of service interfaces. These interface descriptions can be used to generate interaction stubs and to dynamically interact with sensors. It is envisioned that this will primarily be done by gateway devices, which continuously search for nearby sensors. In static environments, service descriptions can be accessed during a deployment process. The information contained in WASP service descriptions about the interface and extra-functional aspects of a service is then used to decide where to deploy code.

A TinyOS implementation of the WASP service description model was implemented [12]. The next steps of WASP development will include integrating the service description model with a service discovery framework and an event-based programming model. This will form the basis of a heterogeneous sensor system and enable pervasive monitoring of the elderly. To validate the service model and the system, the proposed framework will be tested and evaluated in testbeds and patient trails. However, for the purpose of this article, we will now focus on the user experience by highlighting some of the parameters that can be observed using the WASP framework. Thus, the focus will be on the elderly care scenarios, namely that of ADL monitoring using wearable and ambient sensors. 


\section{ADL MONITORING USING AMBIENT AND WEARABLE SENSORS}

The use of pervasive sensing for an elderly care scenario can provide a continuous multi-dimensional picture of an elderly person's well-being rather than just capturing snapshots of the person's physiology and current physical parameters. Activities of interest for the elderly include: leaving and returning home, social interaction, preparing food and eating and sleeping. The use of a large number of ambient or wearable sensors to monitor these activities poses problems of repeatability, installation, and patient comfort. Thus, the number of sensors has to be minimised and optimised to provide sufficient information of the elderly person's well-being, without affecting his/her patterns of daily living. Intelligent data analysis can then be used to infer data from multiple sensors, detect patterns across datasets, and identify risks. In addition, multi-sensor fusion can be used to obtain better classification rates and decrease ambiguity between activities.

\section{A. Ambient Sensors}
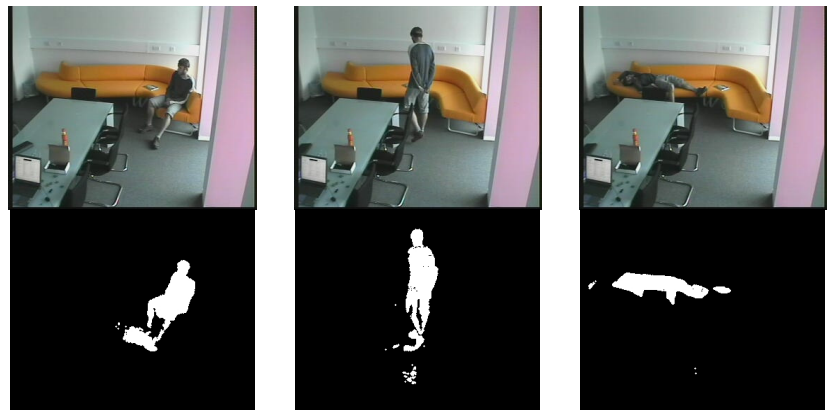

Figure 4. Blob sensor detection of activities showing sitting, walking and lying down.

A recent survey summarizes the current state-of-the-art in smart environments [8], detailing several ambient sensors such as microphones, pressure sensors, RFID tags, electricity and water usage sensors. In [15], a blob sensor, based on the concept of using abstracted image blobs to derive personal metrics and perform behaviour profiling, was proposed. The shape of a blob (or silhouette) captured by the sensor often depends on the relative position of the subject and the sensor. A view-independent model can be generated by fusing a set of blobs captured by respective sensors at different positions, and which can then be used to generate a more detailed activity signature (Fig. 4). Blob sensors can also provide an indication of room occupancy, that can be presented on an activity grid (Fig. 5) and which can be used to observe the activity of the elderly person over time, as well as detect variations in behaviour (as done in [16]), and observe social interaction.

\section{B. Wearable Sensors}

Several groups have investigated the optimal positions for placing wearable sensors, ranging from an arm band [17] to a waist sensor unit [18] and a shoe worn sensor [19]. An ear worn sensor (e-AR sensor) was presented in [20], presenting an unobtrusive technique for measuring activity and physical parameters. The e-AR sensor consists of a 3-axis accelerometer, as well as a Pulse Oximeter for the measurement of oxygen saturation levels $\left(\mathrm{SpO}_{2}\right)$ and heart rate.

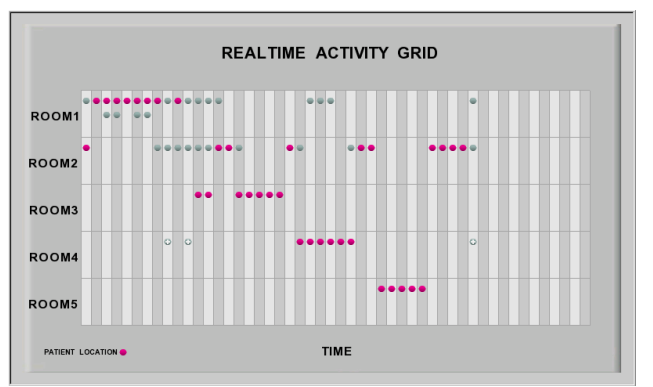

Figure 5. Real-time activity grid showing the elderly person (pink circle) moving between 5 rooms over a period of time. Other people are presented as gray circles. A plus sign is added if more than 2 other people are detected.

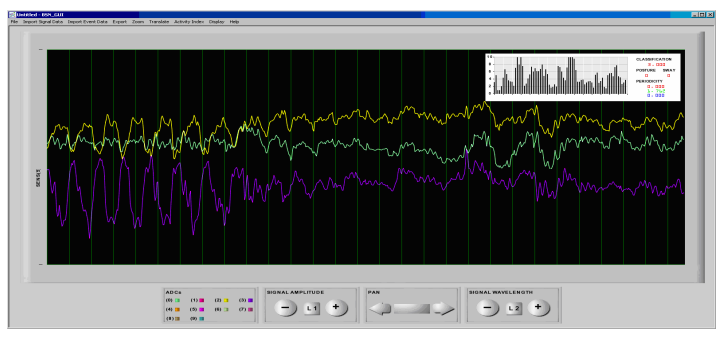

(a)

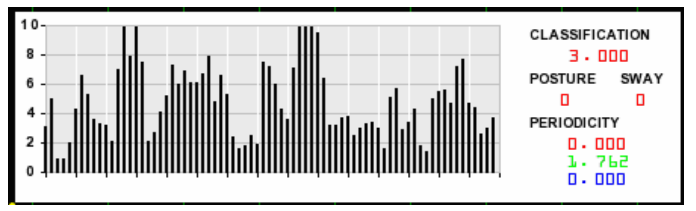

(b)

Figure 6. A user activity display for the e-AR sensor, showing the acceleration signals in (a) and the classification, posture change, swaying and periodicity in (b).

Fig. 6 shows a graphical user interface for the online display of the e-AR sensor signals. The panel on the right of Fig. 6 (a), magnified in Fig. 6(b), shows a real-time bar-chart of the derived activity level (or activity index), which is an averaged variance over the 3 acceleration axes. In addition to that, a classifier that was presented in [20] is used to show the activity class (classified broadly into very low level, low level, medium and high level activities). Detection of sway, posture change and periodicity are also inferred based on the accelerometer signals. A swaying motion could be the precursive to a fall, whereas a sudden change of posture could indicate a fall happening in real-time. These measurements could prove to be very useful in monitoring the elderly who live alone. The activity index could be of use when the decline or improvement in the activity of the elderly needs to be observed over time, and can be viewed in conjunction to the $\mathrm{SpO}_{2}$ and heart rate in order to obtain a clearer picture of the relationship between activity and physical parameters (as in Fig.7). A recent study [21] has shown how this activity index can be used to observe post-operative patients over a few days. Fig. 8 shows a comparison of activity levels for the same period of time over a few days before and after an operation. A similar analysis can be applied to facilitate elderly care. 


\section{Sensor Fusion}

Using information from both ambient and wearable sensors could solve several problems that can not be dealt with by simply using each modality on its own. Classification rates between different activities, for example, can be improved significantly when information from both the ambient and wearable sensors is used, as shown in [15]). Patient identification in the presence of several people can also be done when both modalities are used, as shown in the pervasive behaviour profiling study [16]. In addition, sensor fusion can address missing and erroneous data which could easily occur in a real-life scenario.
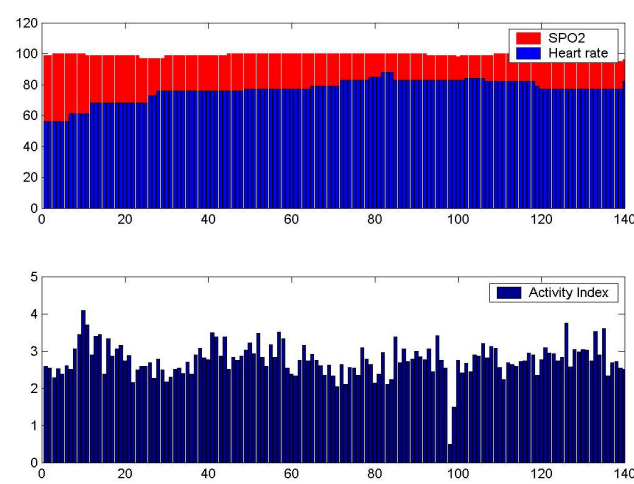

Figure 7. A graph showing the change of $\mathrm{SPO} 2$ and heart rate as well as the change in activity index while the user is performing a set of activities.

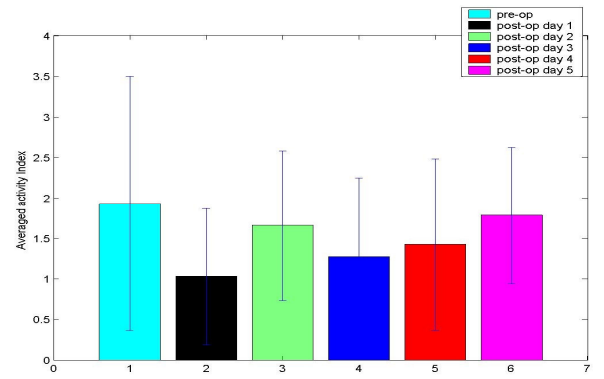

Figure 8. The change of activity levels before and after an operation [21].

\section{CONCLUSIONS}

The WASP service model presents a novel approach to tackle problems such as interoperability, heterogeneity, optimisation and dynamic environments in pervasive sensing scenarios. Integrating this model with an efficient event based programming model is the next challenge in the WASP project. Providing the maximum usability for the elderly and their carers is also a great challenge, as the aim of WASP is to provide a system that is both efficient and easy to use.

\section{ACKNOWLEDGMENT}

This work is supported by the European Commission under the Framework 6, IST Project "Wirelessly Accessible Sensor Populations (WASP). It is in close collaboration with the UK DTI funded SAPHE (Smart and Aware Pervasive Healthcare
Environment) [6] and the UK EPSRC funded BioSensornet projects. Contributions from the following WASP team members are acknowledged: Hannah Wells (Imperial College London), Enrico Frumento (Cefriel), Marco Mazzu (HTN), Laurent Gomez and Annett Laube (SAP).

\section{REFERENCES}

[1] “Aging, can we stop the clock?" Wellcome Trust Report (http://www.wellcome.ac.uk/)

[2] Yang, G.-Z., et al. "From Sensor Networks to Behaviour Profiling: A Homecare Perspective of Intelligent Building." in The IEE Seminar for Intelligent Buildings. 2004: IEE.

[3] P. Bonato. "Advances in wearable technology and applications in physical medicine and rehabilitation." J. Neuroengineering Rehabil., vol. 2, num. 1, pp. 2-6, 2005.

[4] H. Herr and A. Wilkenfeld. "User-adaptive control of a magnetorheological prosthetic knee." Industrial Robot: An International Journal, vol 30. pp. 42-55, 2003.

[5] G. Abowd, A. Bobick, I. Essa, E. Mynatt, and W. Rogers. "The aware home: Developing technologies for successful aging." In Proceedings of AAAI Workshop and Automation as a Care Giver, 2002.

[6] SAPHE Project website http://ubimon.doc.ic.ac.uk/saphe/

[7] T. Tamura. "Biomedical engineering at the forefront in Japan." Engineering in Medicine and Biology Magazine, IEEE, vol. 24, num. 4 pp. 23-26, 2005

[8] D. Cook and S.K. Das. "How smart are our environments? An updated look at the state of the art." Pervasive Mob. Comput., vol. 3, num. 2, pp.53-73, 2007.

[9] "Specified application requirements and prototype selection" - WASP Deliverable $6.2 \mathrm{http}: / / \mathrm{www} . w a s p-p r o j e c t . o r g /$

[10] Yang, G.-Z., "Body Sensor Networks", ed. G.-Z. Yang. 2006, London: Springer-Verlag

[11] "DAML-S Coalition: DAML-S: Web Service Description for the Semantic Web." First International Semantic Web Conference, Italy, 2002.

[12] "Service Model Design for WASP - Deliverable 5.1" http://www.waspproject.org/

[13] EPCglobal: http://www.epcglobal.com

[14] "An overview of IEEE 1451.4 Transducer Electronic Data Sheets (TEDS)", http://standards.ieee.org/regauth/1451

[15] J. Pansiot, D. Stoyanov, D. McIlwraith, B. Lo, and G.Z. Yang. "Ambient and Wearable Sensor Fusion for Activity Recognition in Healthcare Monitoring Systems.” In Proc. of BSN 07, pp. 208-212, 2007.

[16] L. Atallah, M. El Helw, J. Pansiot, J. Stoyanov, L. Wang, and G.Z. Yang. "Behaviour profiling with ambient and wearable sensing." In Proc. of BSN 07, pp. 133-138, 2007

[17] A. Krause, A. Smailagic, and D. Siewiorek. "Context-aware mobile computing: Learning context-dependent personal preferences from a wearable sensor array." IEEE Transactions on Mobile Computing, vol. 5, num. 2, pp.113-127, 2006

[18] S.W. Lee and K. Mase. "Activity and location recognition using wearable sensors." IEEE Pervasive Computing, vol. 1, num.3, pp.24-32, 2002.

[19] S. Morris and J. Paradiso. "Shoe-integrated sensor system for wireless gait analysis and real-time feedback." In Proceedings of the 2nd Joint IEEE EMBS (Engineering in Medicine and Biology Society) and BMES(the Biomedical Engineering Society) Conference. (2002), pp. 2468-2469, 2002.

[20] B. Lo, L. Atallah, O. Aziz, M. ElHelw, A. Darzi, and G-Z. Yang. "Realtime pervasive monitoring for postoperative care." In Proc. of BSN07, volume 1 of IFMBE, pp. 122-127, 2007.

[21] O. Aziz, L. Atallah, B. Lo, M. ElHelw, L. Wang, G.Z. Yang, and A. Darzi. "A Pervasive Body Sensor Network for Measuring Postoperative Recovery at Home." Surgical Innovation, vol. 14, num. 2, pp. 83-90, 2007. 